\title{
An Apple Extract Beverage Combined with Caffeine Can Improve Alertness, Mental Fatigue, and Information Processing Speed
}

\author{
Christie L. Ward-Ritacco ${ }^{1}$ • Alissa R. Wilson ${ }^{2}$ • Patrick J. O'Connor ${ }^{3}$ \\ Received: 17 June 2020 / Accepted: 9 December 2020 / Published online: 4 January 2021 \\ (C) The Author(s) 2021
}

\begin{abstract}
The psychological effects of low-dose caffeine combined with polyphenols from apples have rarely been explored scientifically yet synergistic effects are plausible. A randomized, double-blind, placebo-controlled cross-over experiment was used to test the psychological effects of apple extract beverages combined with 10, 20, 37.5, and $75 \mathrm{mg}$ caffeine. Comparisons were made to both a placebo drink that was artificially sweetened and colored to mimic the test beverages and a positive control drink with $75 \mathrm{mg}$ caffeine but without apple extract. Compared to placebo, it was hypothesized that dose-dependent improvements in cognitive performance, mood, and motivation would be realized after consuming the beverage with apple extract containing added caffeine. Outcomes were assessed before, 60 to 110, and 125 to $175 \mathrm{~min}$ post-beverage. The positive control beverage resulted in more serial seven subtractions, greater motivation to perform cognitive tasks, and reduced feelings of fatigue (all $p<.005$ ). The study found that psychological effects (i) were not observed for beverages containing apple extract and 10 or $20 \mathrm{mg}$ caffeine, (ii) of the apple extract beverage containing $75 \mathrm{mg}$ caffeine generally mimicked the effects of the positive control drink and significantly increased serial seven processing speed, and (iii) of the apple extract beverage containing $37.5 \mathrm{mg}$ improved feelings of alertness and mental fatigue. In sum, effects of apple extract combined with caffeine were not dosedependent; the apple extract beverage containing $75 \mathrm{mg}$ caffeine improved information processing speed and the apple extract beverage with $37.5 \mathrm{mg}$ caffeine improved feelings of alertness and mental fatigue.
\end{abstract}

Keywords Attention $\cdot$ Caffeine $\cdot$ Human cognition $\cdot$ Memory $\cdot$ Motivation

\section{Introduction}

Many people are interested in increasing mental energy, which has been conceptualized as enhancing self-motivation, optimizing cognitive performance, and combating daily feelings of fatigue (O'Connor 2006). One proven short-term strategy toward these ends is the consumption of tea, coffee, cola, and energy drinks, all of which contain caffeine. Psychostimulant effects of caffeine occur because caffeine crosses the blood-brain barrier, antagonizes adenosine receptors, and increases the release of neurotransmitters, such as

Patrick J. O'Connor

poconnor@uga.edu

1 Department of Kinesiology, University of Rhode Island, Kingston, RI 02881, USA

2 Nutrition Sciences, PepsiCo R\&D, Purchase, NY 10577, USA

3 Department of Kinesiology, University of Georgia, Room 115-L, 330 River Road, Athens, GA 30602-6554, USA dopamine and norepinephrine, in brain circuits involved in cognition, mood, and motivation (Fredholm 2012). Concerns have been raised about the potential disadvantages of too much caffeine consumption, and common side effects of large doses of caffeine among susceptible individuals include anxiety, cardiac symptoms, and insomnia (Gonzalez de Mejia and Ramirez-Mares 2014). Increased attention is being paid to understanding the potential psychological benefits of ingesting ingredients other than caffeine, either alone or in combination with smaller amounts of caffeine that are typically consumed.

A small body of research has investigated the psychological consequences of products made from plants that are rich in polyphenols. Polyphenols are naturally occurring chemicals in tea, coffee, chocolate, wine, vegetables, and fruits that can have acute positive effects on cognitive performance, mood, and motivation (Lamport et al. 2012). Most investigations into the mental effects of polyphenols have examined the acute effects of cocoa consumption (Field et al. 2011; Pase et al. 2013; Scholey et al. 2010). The per capita consumption of 
apples is higher than cocoa or other fruits that have been studied. Apples are a major source of dietary polyphenols, yet the psychological consequences of acute apple, or apple juice, consumption have been studied infrequently.

Though inadequately understood, it appears biologically plausible that bioactive constituents from apple juice could act on the brain and cause psychological and behavioral effects. Several polyphenols are detectable in the blood after human consumption of apple juice including caffeic acid, phloretin, (+)-catechin, (-)-epicatechin, and quercetin (Kahle et al. 2011). In rodents, caffeic acid phenethyl esters can enhance cerebral blood flow and bind to central nervous system benzodiazepine receptors (Khan et al. 2007; Marder et al. 1996). In chicks, phloretin acutely inhibits memory for an unpleasant taste by blocking glucose transport (Gibbs et al. 2008). In humans, consumption of an extract containing primarily epigallocatechin gallate resulted in short-term increased electroencephalographic activity and increased ratings of calmness (Scholey et al. 2012). In humans, apple consumption may have effects similar to those of tea or cocoa which increase brain blood flow, perhaps by activating the nitric oxide system, and can enhance mood and aspects of cognitive performance (Fisher et al. 2006; Wightman et al. 2012). One study of middle-aged Australian adults reported that, even though nitric oxide was augmented, there were no acute effects on mood or cognitive performance of consuming $120 \mathrm{~g}$ of raw or cooked homogenized Cripps Pink apple skin and flesh compared to a control consisting of apple flesh alone (which contained low amounts of polyphenols) (Bondonno et al. 2014). The polyphenolic content of apple varieties from different countries varies widely (Alonso-Salces et al. 2004; Marks et al. 2007), and the bioavailability of polyphenols or their effects on cognitive performance may be moderated by age (Chen and Blumberg 2008).

Epicatechin-rich apple extract (Evesse) increases brachial artery blood flow in hypertensive adults (Saarenhovi et al. 2017). Currently unknown is whether this epicatechin-rich apple extract consumption has synergistic effects when combined with caffeine, such that greater psychological benefits could be obtained from drinks containing low doses of caffeine (e.g., $<20 \mathrm{mg}$ ) through the addition of apple extract. The potential synergistic effects are predicated on caffeine working via adenosine receptor antagonism and apple extract causing psychological effects via one of the non-adenosine mechanisms suggested above. Thus, the primary purpose of the research summarized here was to test the short-term psychological effects of consuming $10 \mathrm{oz}$ of apple extract (containing $275 \mathrm{mg}$ of polyphenols) combined with varying amounts of caffeine. Acute cognitive, mood, and motivation benefits of caffeine are often realized after consuming caffeine doses from 37.5 to $450 \mathrm{mg}$; however, one study showed beneficial effects with only $12.5 \mathrm{mg}$ caffeine (Smit and Rogers 2000). We hypothesized that in comparison to a colored and flavored placebo beverage without apple extract or caffeine, cognitive performance, mood, and motivation would improve after consuming apple extract containing added caffeine in low doses of 10 and $20 \mathrm{mg}$ and the extent of the psychological improvements was expected to be greater for apple extract drinks containing larger doses of added caffeine (37.5 and $75 \mathrm{mg}$ ).

\section{Materials and Methods}

\section{Study Design}

A randomized, double-blind, placebo-controlled, cross-over experiment was conducted. Participants completed a series of six test sessions and consumed one of six test beverages during each session. Test beverages were manufactured in 3 batches to minimize storage-related reductions in polyphenolic content (PepsiCo, Valhalla, NY), poured into 10-oz plastic bottles, labelled with a three-digit code number, and shipped to Athens, GA, where they were refrigerated at $\sim 3{ }^{\circ} \mathrm{C}$ until consumed. The apple extract was a natural extract derived from Evesse apples supplied by Coressence, Ltd. and sold by Danisco as Evesse ${ }^{\mathrm{TM}} \mathrm{EPC}$. A detailed description of the extraction method and characterization of the nutrient composition has been published (Saarenhovi et al. 2017). All beverages were consumed within 9 weeks of being manufactured and contained water, sucrose, citric acid, flavoring, colors (caramel and grape blue), sodium hexametophophate, potassium benzoate, calcium disodium EDTA, sucralose, potassium citrate, and acesulfame potassium. The placebo, labelled test beverage 1 , contained no caffeine or apple extract. The positive control, test beverage 2, contained $75 \mathrm{mg}$ of caffeine and no apple extract. Each of the other beverages contained apple extract standardized to contain $275 \mathrm{mg}$ total polyphenols ( $90 \%$ total polyphenols as catechin equivalents) and varying amounts of caffeine: test beverage $3(75 \mathrm{mg})$, test beverage $4(37.5 \mathrm{mg})$, test beverage $5(20 \mathrm{mg})$, and test beverage $6(10 \mathrm{mg})$. Other comparisons of potential interest, such as apple extract alone, were not included to minimize dropout likely to result from the additional test sessions and avoid adding further to the substantial subject burden.

\section{Participants}

The study was approved by the University Institutional Review Committee for human research participants. Participants were recruited via emails to campus listservs and by flyers placed on bulletin boards across the University campus. Participants were compensated up to a total of $\$ 180.00$ for participation in the study ( $\$ 30.00$ for each completed study session). 


\section{Online Screening}

Participants completed online screening questionnaires to determine initial eligibility. This included reporting typical daily caffeine consumption, sensitivity to caffeine, medication use, demographics, and health status indicators including history of psychological disorder(s), history of heart rhythm disorder(s), and daily physical activity level as measured by the Godin Leisure Time Exercise questionnaire (Godin and Shephard 1985).

\section{In-person Screening Visit}

Upon successful completion of the online screening, eligible participants were invited to participate in an on-site laboratory screening visit. This visit consisted of confirming eligibility, completing a sleep questionnaire, and completing a subset of the cognitive and mood measures to ensure understanding of the instructions and testing procedures, as well as to minimize practice effects and exclude those potential participants who were unable or unwilling to perform adequately on the cognitive tests. An approved informed consent document, consistent with the Declaration of Helsinki, was signed by all participants during this visit.

\section{Inclusion/Exclusion Criteria}

Participants were excluded if they (a) were younger than 18 years old or older than 49 years of age, (b) were currently using prescription medication, (c) reported high sensitivity to caffeine, (d) reported diagnosis of any heart rhythm disorders, (d) reported high levels of habitual caffeine use $(>800 \mathrm{mg}$ caffeine per day; equivalent to $\sim 8$ cups of coffee), (e) reported a history of a psychological disorder, and/or ( $g$ ) did not demonstrate adequate proficiency in the cognitive tasks during the screening visit.

\section{Sample Size Calculation}

An a priori sample size was determined using a statistical power calculation (D'Amico et al. 2001). The calculation assumed a two-tailed alpha value of 0.05 and a correlation between repeated measures of $r=0.80$. A sample of 25 participants was adequately powerful $(1-\beta=0.80)$ to detect a moderate-sized, practically meaningful condition $\times$ time interaction effect expressed as partial eta-squared $\left(\eta^{2}\right.$ of $\left.\geq 0.06\right)$.

\section{Final Sample}

The flow of participants through the trial is presented in Fig. 1. One hundred and fifty-two individuals contacted the laboratory and initiated the initial online screening process. Eightyeight individuals who completed the initial screening questionnaire were eligible for in-person screening. Of these 88,45 completed the screening visit, and the remaining 43 were lost to follow-up or declined to participate in the inperson screening. Fifteen individuals who completed the inperson screening process declined to enroll, while 30 participants were enrolled and completed at least one experimental study visit. Five participants were excluded from final analysis due to incomplete data ( $n=4$, time commitment too great) and protocol non-compliance $(n=1)$. Thus, 25 healthy volunteers (14 females and 11 males) completed the full protocol.

\section{Experimental Testing Sessions}

Participants were asked to eat a similar meal and snack before arriving to each day's testing session and refrain from consuming apples and foods or beverages containing apples from the time they woke up until the time they arrived for their testing sessions. Upon arriving for testing, the participants completed a food log detailing their morning meals/snacks, confirmed that they had a typical night's sleep $( \pm 2 \mathrm{~h}$ from usual), and abstained from both caffeine and structured exercise from the time they woke up until they arrived for their scheduled testing session. Each participant was tested at approximately the same time of day across the six beverage conditions but across participants the time of day of testing varied (e.g., some individuals were tested in the morning and others were tested in the afternoon).

Figure 2 summarizes the structure of the testing sessions. Test beverage assignments for each visit were randomized for all participants. Participants first completed baseline testing, which took 50 to $55 \mathrm{~min}$, and were then provided with their test beverage and given $15 \mathrm{~min}$ to consume it. This was followed by a 60 -min rest period, to provide time for the beverage ingredients to become bioactive. During this time, the participants were allowed bathroom use and water. Also, they could watch a nature documentary, read, or rest. Participants completed the same activity during the break at each visit. Next, post test beverage 1 testing was completed, followed by a 15 -min break in which the participant walked $30 \mathrm{ft}$ to an adjacent room and sat quietly. This was followed by the final post test beverage 2 testing. All testing sessions were conducted in a sound-dampened, isolated testing chamber.

\section{Cognitive Demand Battery}

The Cognitive Demand Battery (CDB), delivered using the COMPASS software program (Northumbria University, Newcastle upon Tyne), has been found to be sensitive to several nutritional experimental manipulations (Wightman et al. 2012). The CDB was administered using a Dell PC (Dell Optiplex 980) with participants seated in a chair with their gaze directed at a 15 -in. computer screen. The CDB was modified in that motivation and Mental and Physical State Energy 


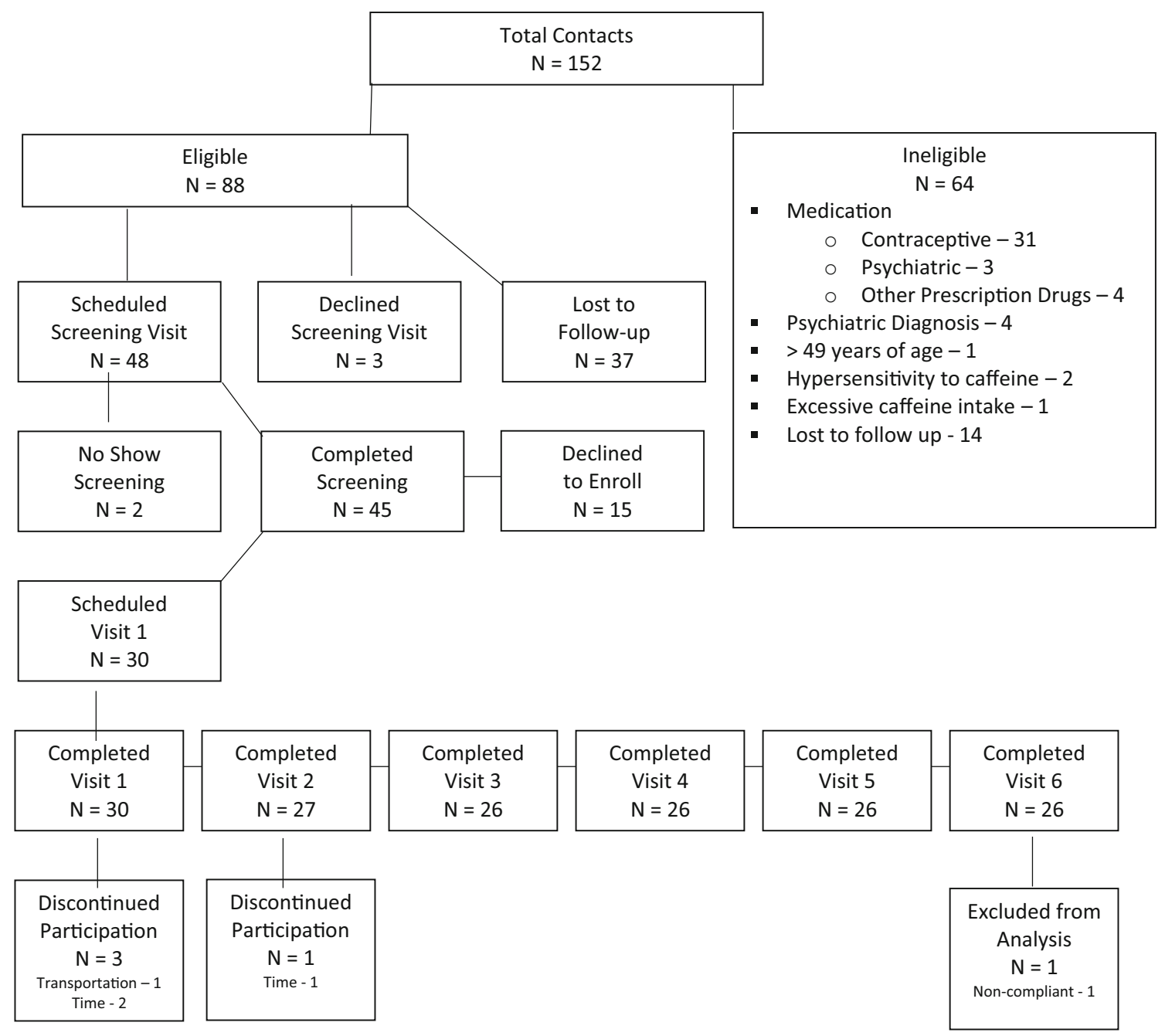

Fig. 1 Participant flow

and Fatigue Scales were added. Cognitive performance was measured with word presentation/recall/recognition, picture presentation/recognition, serial 3 and serial 7 subtraction tasks, and rapid visual information processing (RVIP) task, while mood and motivation were measured with visual analog scales (Maridakis et al. 2009).
Fig. 2 Structure of testing sessions. Total testing time ranged from 4 to $4.25 \mathrm{~h}$. RVIP rapid visual information processing
1. Baseline - Cognitive, Mood and Motivation Assessments (50-55 minutes)

Motivation (15 seconds)

Mood (60 seconds)

Cognitive Demand Battery

Word Presentation (15 seconds) -> Picture Presentation (45 seconds)

Serial Threes (2 min) -> Serial Sevens (2 min) -> RVIP $(5 \mathrm{~min})$-> Mood and Motivation Assessment (2-3 min) Serial Threes $(2 \mathrm{~min})->$ Serial Sevens $(2 \mathrm{~min}) \rightarrow$ RVIP $(5 \mathrm{~min}) \rightarrow$ Mood and Motivation Assessment $(2-3 \mathrm{~min})$ Serial Threes (2 min) -> Serial Sevens (2 min) -> RVIP (5 min) -> Mood and Motivation Assessment (2-3 min)

Serial Threes (2 min) -> Serial Sevens (2 min) -> RVIP (5 min) -> Mood and Motivation Assessment (2-3 min) Word Recall (1 min) -> Word Recognition (30 seconds) -> Picture Recognition (30 seconds)

\section{Treatment (up to 15 minutes}

3. Quiet, seated rest break (60 minute)

4. Post Treatment 1 - Cognitive, mood and Motivation Assessments (50-55 minutes)

5. Quiet, seated rest break (15 minutes)

6. Post Treatment 2 - Cognitive, Mood and Motivation Assessments (50-55 minutes) 


\section{Data Analysis}

All analyses were performed using SPSS v22 (IBM SPSS, Armonk, NY). Preliminary analyses included intraclass correlations (ICC) that examined whether the cognitive performance, mood, and motivation scores measured at baseline were reliable. The ICC model used was a two-way ( 25 participants and 4 baseline trials) mixed effects model (participants were considered random and trials fixed effects). The ICC model used absolute agreement for single measures. Reliability was adequately high for the motivation (ICC = 0.792), mood (mean ICC $=0.836$ ), and cognitive outcomes that involved performance accuracy (mean ICC $=.841$ ). Reliability was low for the cognitive performance error measures (mean ICC $=0.292$ ) and RVIP reaction time $(\mathrm{ICC}=$ 0.513). The mean of four values for each outcome variable served as the criterion measure at each of the three time points (BL, post-beverage 1, and post-beverage 2).

The primary analysis involved analysis of variance (ANOVA); specifically a series of two-way ANOVAs: 2 Beverages (placebo and test beverage) X 3 Times (baseline, post-beverage 1, and post-beverage 2). Adjustments for sphericity, when needed, were made using Huynh-Feldt epsilon. Hypotheses were focused on the presence of statistically significant Beverage x Time interactions. Main effects of Beverage and Time were not of interest. The magnitude of the effects from the ANOVAs is provided as partial etasquared $\left(\eta_{p}{ }^{2}\right)$. To control for error associated with multiple statistical tests, $p$ values $\leq .005$ were judged as statistically significant. Significant interactions were followed up by one-way ANOVAs and $t$-tests to determine if changes in the test beverage increased at post-beverage 1 or post-beverage 2 compared to baseline. Significant interactions also were followed up with ANCOVAs which tested if the findings were explained by adding typical caffeine consumption as a covariate.

\section{Results}

No adverse events occurred for any of the beverage doses used in the present study.

\section{Participants}

Twenty-five participants ( 14 female, 11 males) had a mean ( \pm SD) age of 24 years ( \pm 6 ; range $18-46$ years), weight of $78.9 \pm 20.53 \mathrm{~kg}$, height of $171.6 \pm 8.4 \mathrm{~cm}$, body mass index of $26.7 \pm 5.8 \mathrm{~kg} \mathrm{~m}^{-2}$, and typical daily caffeine consumption of $205.6 \pm 208.4 \mathrm{mg}$ (range $0-741.0 \mathrm{mg}$ ). Participants reported strenuous physical activity scores of $31.7 \pm 17.5$ (range 0-63), moderate physical activity scores of $18.8 \pm$ 10.9 (range $0-35$ ), and low physical activity scores of 11.2 \pm 8.9 (range $0-30$ ). The mean total score of 62 is consistent with total Godin physical activity scores previously reported for groups of college students (Reed and Phillips 2005). Mean sleep durations the night before testing did not differ among the conditions $(p=.958)$ : beverage $1(7.26 \pm$ $0.97 \mathrm{~h})$, beverage $2(7.28 \pm 0.93 \mathrm{~h})$, beverage $3(7.36 \pm$ $1.07 \mathrm{~h})$, beverage $4(7.18 \pm 1.09 \mathrm{~h})$, beverage $5(7.26 \pm$ $0.87 \mathrm{~h})$, and beverage $6(7.10 \pm 0.91 \mathrm{~h})$.

\section{Outcomes for Cognitive Performance and Motivation to Perform the Cognitive Tasks}

Descriptive statistics for the cognitive performance outcomes are provided in Table 1 . The statistically significant results for cognitive performance and motivation outcomes are summarized below, and outcomes not mentioned below were statistically insignificant $(p>.005)$.

Positive Control (75 mg Caffeine Condition) The largest effects on cognitive performance were found for the serial subtraction tasks. There was a significant Group $\mathrm{x}$ Time interaction for $75 \mathrm{mg}$ caffeine condition (CAF) for the total number of attempted serial 3 and serial 7 subtractions $\left(\eta_{p}{ }^{2}=.122\right.$, $p=.003$ and $\eta_{p}{ }^{2}=.171, p<.001$, respectively). Post hoc tests showed that serial 3 and 7 total number of subtractions attempted was increased compared to baseline at both postbeverage time points. There was a significant Group $\mathrm{x}$ Time interaction for $75 \mathrm{mg}$ CAF for the accuracy of both serial 3 and serial 7 subtractions $\left(\eta_{p}{ }^{2}=.102, p=.005 ; \eta_{p}{ }^{2}=.146\right.$, $p<.001$, respectively). Post hoc tests showed that serial 3 and 7 accuracy was increased compared to baseline at both post-beverage time points. The positive control beverage also significantly increased self-reported motivation to perform the cognitive tasks $\left(\eta_{p}{ }^{2}=.121, p=.004\right)$ at both post-beverage time points.

$75 \mathrm{mg}$ CAF + Apple Extract There was a significant Group $\mathrm{x}$ Time interaction for $75 \mathrm{mg} \mathrm{CAF}+$ apple extract for the total number of serial 7 subtractions attempted $\left(\eta_{p}{ }^{2}=.083\right.$, $p<.001)$. Post hoc tests showed that the number attempted increased compared to baseline at both post-beverage time points. The number attempted was increased compared to baseline by a mean $( \pm \mathrm{SD})$ of $4.1( \pm 4.5)$ and $5.4( \pm 4.3)$ at the first and second post-beverage time points, respectively.

$37.5 \mathrm{mg}$ CAF + Apple Extract The Group x Time interactions for $37.5 \mathrm{mg} \mathrm{CAF}+$ apple extract effects on cognition were all statistically insignificant but several were in the expected direction, including an increased total number of attempted serial 3 subtractions ( $p=.01$ ), an increased total number of correct serial 7 subtractions $(p=.049)$, and faster reaction time during correct RVIP responses $(p=.031)$. The beverage also tended to increase self-reported motivation to perform the 
Table 1 Mean $( \pm$ SD) scores for motivation and cognitive performance variables across beverage conditions and time, and interaction significance

\begin{tabular}{|c|c|c|c|c|c|c|c|c|}
\hline & & \multicolumn{2}{|c|}{ Baseline (BL) } & \multicolumn{2}{|c|}{$\begin{array}{l}\text { Post } 1 \\
\text { Change from } \mathrm{BL}\end{array}$} & \multicolumn{2}{|c|}{$\begin{array}{l}\text { Post } 2 \\
\text { Change from BL }\end{array}$} & \multirow[t]{2}{*}{$\begin{array}{l}\text { Beverage } \mathrm{x} \text { Time }^{\mathrm{a}} \\
p \text { value }\end{array}$} \\
\hline & & Value & $\mathrm{SD}$ & Value & SD & Value & $\mathrm{SD}$ & \\
\hline \multirow{6}{*}{$\begin{array}{l}\text { Motivation to perform cognitive tests } \\
\text { (VAS within CDB) }\end{array}$} & Placebo & 43.99 & 17.09 & -1.15 & 13.36 & -1.95 & 19.30 & \\
\hline & $75.0 \mathrm{mg} \mathrm{C}$ & 41.01 & 19.93 & 14.09 & 15.91 & 10.08 & 18.23 & .004 \\
\hline & $75.0 \mathrm{mg} \mathrm{C}+\mathrm{AJ}$ & 43.46 & 20.03 & 6.1 & 17.26 & 3.85 & 18.79 & \\
\hline & $37.5 \mathrm{mg} \mathrm{C}+\mathrm{AJ}$ & 40.08 & 16.25 & 11.91 & 21.05 & 9.99 & 20.21 & \\
\hline & $20.0 \mathrm{mg} \mathrm{C}+\mathrm{AJ}$ & 46.01 & 17.23 & 3.75 & 11.75 & -0.23 & 12.88 & \\
\hline & $10.0 \mathrm{mg} \mathrm{C}+\mathrm{AJ}$ & 39.49 & 18.23 & 4.52 & 19.74 & 7.70 & 21.42 & \\
\hline \multirow{6}{*}{$\begin{array}{l}\text { Serial threes - accuracy } \\
\text { (number) }\end{array}$} & Placebo & 51.48 & 18.96 & 1.72 & 7.77 & 1.26 & 10.24 & \\
\hline & $75.0 \mathrm{mg} \mathrm{C}$ & 48.75 & 15.47 & 6.28 & 5.25 & 7.68 & 5.75 & \\
\hline & $75.0 \mathrm{mg} \mathrm{C}+\mathrm{AJ}$ & 52.12 & 16.57 & 4.16 & 6.28 & 4.77 & 4.86 & \\
\hline & $37.5 \mathrm{mg} \mathrm{C}+\mathrm{AJ}$ & 46.66 & 17.26 & 4.71 & 5.24 & 6.64 & 8.20 & \\
\hline & $20.0 \mathrm{mg} \mathrm{C}+\mathrm{AJ}$ & 53.77 & 18.39 & -0.12 & 8.25 & -0.11 & 6.70 & \\
\hline & $10.0 \mathrm{mg} \mathrm{C}+\mathrm{AJ}$ & 48.40 & 14.65 & 1.47 & 7.25 & 4.18 & 8.08 & \\
\hline \multirow{6}{*}{$\begin{array}{l}\text { Serial three - errors } \\
\text { (number) }\end{array}$} & Placebo & 2.47 & 2.36 & -0.23 & 2.33 & -0.63 & 2.40 & \\
\hline & $75.0 \mathrm{mg} \mathrm{C}$ & 2.28 & 1.99 & -0.29 & 1.68 & -0.21 & 1.75 & \\
\hline & $75.0 \mathrm{mg} \mathrm{C}+\mathrm{AJ}$ & 2.46 & 1.81 & -0.01 & 1.39 & -0.49 & 1.33 & \\
\hline & $37.5 \mathrm{mg} \mathrm{C}+\mathrm{AJ}$ & 2.22 & 21.47 & -0.07 & 1.43 & 0.07 & 1.83 & \\
\hline & $20.0 \mathrm{mg} \mathrm{C}+\mathrm{AJ}$ & 2.38 & 1.92 & -0.18 & 1.53 & 0.15 & 1.53 & \\
\hline & $10.0 \mathrm{mg} \mathrm{C}+\mathrm{AJ}$ & 2.63 & 1.61 & -0.35 & 1.51 & 0.23 & 2.62 & \\
\hline \multirow{6}{*}{$\begin{array}{l}\text { Serial sevens - total attempted } \\
\text { (number) }\end{array}$} & Placebo & 36.53 & 16.89 & 0.46 & 4.89 & 3.13 & 5.18 & \\
\hline & $75.0 \mathrm{mg} \mathrm{C}$ & 33.22 & 13.83 & 5.58 & 4.05 & 7.66 & 5.35 & $<.001$ \\
\hline & $75.0 \mathrm{mg} \mathrm{C}+\mathrm{AJ}$ & 35.10 & 14.19 & 4.05 & 4.45 & 5.42 & 4.28 & \\
\hline & $37.5 \mathrm{mg} \mathrm{C}+\mathrm{AJ}$ & 32.58 & 16.67 & 2.79 & 5.48 & 6.16 & 5.04 & \\
\hline & $20.0 \mathrm{mg} \mathrm{C}+\mathrm{AJ}$ & 36.72 & 16.84 & 2.22 & 4.37 & 2.58 & 3.64 & \\
\hline & $10.0 \mathrm{mg} \mathrm{C}+\mathrm{AJ}$ & 31.77 & 12.40 & 3.99 & 3.88 & 4.32 & 5.47 & \\
\hline \multirow{6}{*}{$\begin{array}{l}\text { Serial sevens - accuracy } \\
\text { (number) }\end{array}$} & Placebo & 34.22 & 17.20 & 0.65 & 4.95 & 2.83 & 5.32 & \\
\hline & $75.0 \mathrm{mg} \mathrm{C}$ & 30.86 & 14.50 & 5.43 & 4.78 & 7.32 & 5.59 & .001 \\
\hline & $75.0 \mathrm{mg} \mathrm{C}+\mathrm{AJ}$ & 32.88 & 14.48 & 4.01 & 5.17 & 5.48 & 4.35 & \\
\hline & $37.5 \mathrm{mg} \mathrm{C}+\mathrm{AJ}$ & 30.02 & 16.69 & 3.06 & 5.65 & 6.22 & 5.33 & \\
\hline & $20.0 \mathrm{mg} \mathrm{C}+\mathrm{AJ}$ & 34.53 & 17.04 & 1.38 & 5.24 & 1.34 & 5.81 & \\
\hline & $10.0 \mathrm{mg} \mathrm{C}+\mathrm{AJ}$ & 28.64 & 12.87 & 3.81 & 4.13 & 4.78 & 5.16 & \\
\hline \multirow{6}{*}{$\begin{array}{l}\text { Serial sevens - errors } \\
\text { (number) }\end{array}$} & Placebo & 2.31 & 1.79 & -0.19 & 1.50 & 0.29 & 1.60 & \\
\hline & $75.0 \mathrm{mg} \mathrm{C}$ & 2.36 & 2.03 & 0.15 & 1.39 & 0.35 & 1.33 & \\
\hline & $75.0 \mathrm{mg} \mathrm{C}+\mathrm{AJ}$ & 2.22 & 1.35 & 0.04 & 1.64 & -0.06 & 1.27 & \\
\hline & $37.5 \mathrm{mg} \mathrm{C}+\mathrm{AJ}$ & 2.56 & 1.63 & -0.27 & 0.87 & -0.07 & 1.81 & \\
\hline & $20.0 \mathrm{mg} \mathrm{C}+\mathrm{AJ}$ & 2.19 & 1.32 & 0.84 & 2.70 & 1.25 & 3.73 & \\
\hline & $10.0 \mathrm{mg} \mathrm{C}+\mathrm{AJ}$ & 3.13 & 2.35 & 0.18 & 2.28 & -0.47 & 1.39 & \\
\hline \multirow{6}{*}{$\begin{array}{l}\mathrm{RVIP}^{\mathrm{b}} \text { - accuracy } \\
(\%)\end{array}$} & Placebo & 39.33 & 18.99 & -1.42 & 6.62 & -1.16 & 10.91 & \\
\hline & $75.0 \mathrm{mg} \mathrm{C}$ & 37.90 & 15.72 & 6.35 & 8.97 & 3.02 & 11.52 & \\
\hline & $75.0 \mathrm{mg} \mathrm{C}+\mathrm{AJ}$ & 39.13 & 19.45 & 1.68 & 10.70 & -0.12 & 11.18 & \\
\hline & $37.5 \mathrm{mg} \mathrm{C}+\mathrm{AJ}$ & 42.75 & 19.09 & 1.4 & 11.03 & -0.32 & 10.97 & \\
\hline & $20.0 \mathrm{mg} \mathrm{C}+\mathrm{AJ}$ & 44.93 & 20.12 & -0.88 & 9.82 & -1.50 & 14.12 & \\
\hline & $10.0 \mathrm{mg} \mathrm{C}+\mathrm{AJ}$ & 42.03 & 17.50 & -0.88 & 12.79 & -1.30 & 11.65 & \\
\hline \multirow{4}{*}{$\begin{array}{l}\mathrm{RVIP}^{\mathrm{b}} \text { - correct response time } \\
(\mathrm{msec})\end{array}$} & Placebo & 539.91 & 45.23 & 0.58 & 24.27 & 8.20 & 39.54 & \\
\hline & $75.0 \mathrm{mg} \mathrm{C}$ & 547.09 & 39.33 & -7.65 & 25.89 & -0.80 & 26.62 & \\
\hline & $75.0 \mathrm{mg} \mathrm{C}+\mathrm{AJ}$ & 543.46 & 40.10 & -15.56 & 53.35 & 5.12 & 28.21 & \\
\hline & $37.5 \mathrm{mg} \mathrm{C}+\mathrm{AJ}$ & 556.19 & 44.19 & -15.38 & 48.17 & -21.04 & 47.93 & \\
\hline
\end{tabular}


Table 1 (continued)

\begin{tabular}{|c|c|c|c|c|c|c|c|c|}
\hline & & \multicolumn{2}{|c|}{ Baseline (BL) } & \multicolumn{2}{|c|}{$\begin{array}{l}\text { Post } 1 \\
\text { Change from BL }\end{array}$} & \multicolumn{2}{|c|}{$\begin{array}{l}\text { Post } 2 \\
\text { Change from BL }\end{array}$} & \multirow[t]{2}{*}{$\begin{array}{l}\text { Beverage } \mathrm{x} \text { Time } \\
p \text { value }\end{array}$} \\
\hline & & Value & $\mathrm{SD}$ & Value & SD & Value & SD & \\
\hline \multirow{8}{*}{$\begin{array}{l}\mathrm{RVIP}^{\mathrm{b}} \text { - false alarms } \\
\text { (number) }\end{array}$} & $20.0 \mathrm{mg} \mathrm{C}+\mathrm{AJ}$ & 546.14 & 47.15 & -13.32 & 44.59 & -3.78 & 27.60 & \\
\hline & $10.0 \mathrm{mg} \mathrm{C}+\mathrm{AJ}$ & 538.91 & 56.70 & 1.75 & 20.37 & 6.39 & 26.46 & \\
\hline & Placebo & 3.73 & 5.71 & -0.02 & 2.91 & -1.23 & 4.16 & \\
\hline & $75.0 \mathrm{mg} \mathrm{C}$ & 4.69 & 8.66 & 0.63 & 4.22 & 0.18 & 4.36 & \\
\hline & $75.0 \mathrm{mg} \mathrm{C}+\mathrm{AJ}$ & 4.36 & 7.74 & 1.81 & 4.09 & 0.34 & 2.79 & \\
\hline & $37.5 \mathrm{mg} \mathrm{C}+\mathrm{AJ}$ & 2.57 & 2.85 & -0.01 & 4.75 & -0.15 & 3.84 & \\
\hline & $20.0 \mathrm{mg} \mathrm{C}+\mathrm{AJ}$ & 2.55 & 3.68 & -0.24 & 3.50 & -0.36 & 4.67 & \\
\hline & $10.0 \mathrm{mg} \mathrm{C}+\mathrm{AJ}$ & 5.21 & 6.63 & 0.10 & 5.97 & -2.17 & 7.03 & \\
\hline \multirow{6}{*}{$\begin{array}{l}\text { Delayed recognition of words presented } \\
\text { (\% accuracy) }\end{array}$} & Placebo & 64.00 & 15.75 & -1.87 & 14.34 & 2.67 & 13.47 & \\
\hline & $75.0 \mathrm{mg} \mathrm{C}$ & 67.73 & 18.53 & -7.20 & 15.14 & -1.60 & 18.18 & \\
\hline & $75.0 \mathrm{mg} \mathrm{C}+\mathrm{AJ}$ & 60.27 & 16.01 & 3.73 & 16.11 & 5.07 & 16.47 & \\
\hline & $37.5 \mathrm{mg} \mathrm{C}+\mathrm{AJ}$ & 67.47 & 17.35 & -2.40 & 16.76 & 1.07 & 18.22 & \\
\hline & $20.0 \mathrm{mg} \mathrm{C}+\mathrm{AJ}$ & 65.87 & 16.81 & -7.20 & 15.74 & -0.01 & 17.21 & \\
\hline & $10.0 \mathrm{mg} \mathrm{C}+\mathrm{AJ}$ & 63.47 & 20.01 & -4.00 & 14.27 & 3.47 & 18.67 & \\
\hline \multirow{6}{*}{$\begin{array}{l}\text { Delayed recognition of words presented } \\
\text { (reaction time, msec) }\end{array}$} & Placebo & 1098.48 & 380.32 & 20.21 & 271.54 & -130.25 & 377.96 & \\
\hline & $75.0 \mathrm{mg} \mathrm{C}$ & 1031.46 & 236.64 & 65.32 & 478.42 & 1.18 & 435.13 & \\
\hline & $75.0 \mathrm{mg} \mathrm{C}+\mathrm{AJ}$ & 1140.81 & 442.74 & -62.14 & 352.06 & -117.85 & 513.31 & \\
\hline & $37.5 \mathrm{mg} \mathrm{C}+\mathrm{AJ}$ & 1009.31 & 237.33 & 75.73 & 222.24 & 19.85 & 275.36 & \\
\hline & $20.0 \mathrm{mg} \mathrm{C}+\mathrm{AJ}$ & 1156.47 & 426.37 & -103.53 & 243.57 & -119.35 & 314.99 & \\
\hline & $10.0 \mathrm{mg} \mathrm{C}+\mathrm{AJ}$ & 1051.40 & 278.33 & -10.64 & 311.14 & 12.05 & 394.51 & \\
\hline \multirow{6}{*}{$\begin{array}{l}\text { Delayed recognition of words not presented } \\
\text { (\% accuracy) }\end{array}$} & Placebo & 83.20 & 18.37 & -2.13 & 15.84 & -4.00 & 12.91 & \\
\hline & $75.0 \mathrm{mg} \mathrm{C}$ & 85.60 & 15.60 & -2.13 & 9.57 & -2.13 & 10.31 & \\
\hline & $75.0 \mathrm{mg} \mathrm{C}+\mathrm{AJ}$ & 81.07 & 19.78 & -1.87 & 17.05 & 1.60 & 11.27 & \\
\hline & $37.5 \mathrm{mg} \mathrm{C}+\mathrm{AJ}$ & 86.67 & 15.40 & -5.33 & 12.32 & -1.87 & 10.28 & \\
\hline & $20.0 \mathrm{mg} \mathrm{C}+\mathrm{AJ}$ & 87.20 & 11.85 & -1.60 & 14.69 & -3.73 & 12.03 & \\
\hline & $10.0 \mathrm{mg} \mathrm{C}+\mathrm{AJ}$ & 85.33 & 14.27 & -3.47 & 12.49 & -2.93 & 15.99 & \\
\hline \multirow{6}{*}{$\begin{array}{l}\text { Delayed recognition of words not presented } \\
\text { (reaction time, msec) }\end{array}$} & Placebo & 1102.38 & 319.18 & -79.95 & 269.16 & -52.20 & 381.10 & \\
\hline & $75.0 \mathrm{mg} \mathrm{C}$ & 1089.10 & 288.40 & -55.26 & 220.21 & -46.95 & 248.26 & \\
\hline & $75.0 \mathrm{mg} \mathrm{C}+\mathrm{AJ}$ & 1087.26 & 287.30 & 88.58 & 325.77 & -63.40 & 303.41 & \\
\hline & $37.5 \mathrm{mg} \mathrm{C}+\mathrm{AJ}$ & 1007.26 & 218.48 & 49.03 & 283.69 & 3.11 & 252.64 & \\
\hline & $20.0 \mathrm{mg} \mathrm{C}+\mathrm{AJ}$ & 1091.33 & 299.26 & -17.58 & 294.18 & -52.55 & 307.18 & \\
\hline & $10.0 \mathrm{mg} \mathrm{C}+\mathrm{AJ}$ & 1070.16 & 240.16 & -4.13 & 278.14 & -27.18 & 348.58 & \\
\hline \multirow{6}{*}{$\begin{array}{l}\text { Delayed recognition of pictures presented } \\
\text { (\% correct) }\end{array}$} & Placebo & 81.33 & 16.56 & -1.87 & 11.47 & 0.53 & 12.01 & \\
\hline & $75.0 \mathrm{mg} \mathrm{C}$ & 83.20 & 15.77 & -3.73 & 14.54 & -5.33 & 15.75 & \\
\hline & $75.0 \mathrm{mg} \mathrm{C}+\mathrm{AJ}$ & 83.47 & 12.34 & -1.87 & 10.46 & -4.00 & 13.33 & \\
\hline & $37.5 \mathrm{mg} \mathrm{C}+\mathrm{AJ}$ & 85.60 & 12.72 & -4.53 & 14.24 & -7.20 & 17.31 & \\
\hline & $20.0 \mathrm{mg} \mathrm{C}+\mathrm{AJ}$ & 88.27 & 12.66 & -4.80 & 10.28 & -5.60 & 14.10 & \\
\hline & $10.0 \mathrm{mg} \mathrm{C}+\mathrm{AJ}$ & 84.53 & 13.97 & -7.20 & 17.21 & 1.60 & 14.31 & \\
\hline \multirow{6}{*}{$\begin{array}{l}\text { Delayed recognition of pictures presented } \\
\text { (reaction time, msec) }\end{array}$} & Placebo & 926.71 & 148.61 & -2.42 & 157.15 & 17.72 & 171.78 & \\
\hline & $75.0 \mathrm{mg} \mathrm{C}$ & 917.79 & 131.29 & -17.32 & 142.46 & 34.21 & 296.36 & \\
\hline & $75.0 \mathrm{mg} \mathrm{C}+\mathrm{AJ}$ & 963.50 & 185.21 & -8.67 & 208.20 & -56.59 & 154.04 & \\
\hline & $37.5 \mathrm{mg} \mathrm{C}+\mathrm{AJ}$ & 983.27 & 244.11 & 1.48 & 286.55 & -84.15 & 285.68 & \\
\hline & $20.0 \mathrm{mg} \mathrm{C}+\mathrm{AJ}$ & 880.54 & 103.43 & 45.94 & 150.28 & 11.13 & 120.70 & \\
\hline & $10.0 \mathrm{mg} \mathrm{C}+\mathrm{AJ}$ & 945.56 & 175.06 & 49.50 & 193.16 & -15.53 & 192.19 & \\
\hline \multirow{2}{*}{$\begin{array}{l}\text { Delayed recognition of pictures not presented } \\
\text { (\% correct) }\end{array}$} & Placebo & 91.73 & 12.81 & 0.00 & 13.33 & -6.13 & 13.04 & \\
\hline & $75.0 \mathrm{mg} \mathrm{C}$ & 94.93 & 8.67 & -1.87 & 9.13 & -4.00 & 8.61 & \\
\hline
\end{tabular}


Table 1 (continued)

\begin{tabular}{|c|c|c|c|c|c|c|c|c|}
\hline & & \multicolumn{2}{|c|}{ Baseline (BL) } & \multicolumn{2}{|c|}{$\begin{array}{l}\text { Post } 1 \\
\text { Change from BL }\end{array}$} & \multicolumn{2}{|c|}{$\begin{array}{l}\text { Post } 2 \\
\text { Change from BL }\end{array}$} & \multirow[t]{2}{*}{$\begin{array}{l}\text { Beverage } \mathrm{x} \text { Time }^{\mathrm{a}} \\
p \text { value }\end{array}$} \\
\hline & & Value & SD & Value & $\mathrm{SD}$ & Value & SD & \\
\hline \multirow{10}{*}{$\begin{array}{l}\text { Delayed recognition of pictures not presented } \\
\text { (reaction time, } \mathrm{msec} \text { ) }\end{array}$} & $75.0 \mathrm{mg} \mathrm{C}+\mathrm{AJ}$ & 90.40 & 14.67 & -0.80 & 10.06 & -4.00 & 13.05 & \\
\hline & $37.5 \mathrm{mg} \mathrm{C}+\mathrm{AJ}$ & 92.53 & 9.87 & 0.80 & 8.89 & -2.67 & 11.39 & \\
\hline & $20.0 \mathrm{mg} \mathrm{C}+\mathrm{AJ}$ & 92.53 & 11.11 & -2.40 & 8.14 & -2.40 & 6.35 & \\
\hline & $10.0 \mathrm{mg} \mathrm{C}+\mathrm{AJ}$ & 92.80 & 15.86 & -6.13 & 7.92 & -3.73 & 13.75 & \\
\hline & Placebo & 992.62 & 17.51 & 67.27 & 390.00 & -6.25 & 213.10 & \\
\hline & $75.0 \mathrm{mg} \mathrm{C}$ & 1020.73 & 226.12 & -24.55 & 198.34 & -6.85 & 166.62 & \\
\hline & $75.0 \mathrm{mg} \mathrm{C}+\mathrm{AJ}$ & 1144.64 & 394.48 & -195.75 & 353.55 & -125.36 & 383.37 & \\
\hline & $37.5 \mathrm{mg} \mathrm{C}+\mathrm{AJ}$ & 1059.97 & 224.39 & 0.02 & 325.82 & -76.02 & 222.65 & \\
\hline & $20.0 \mathrm{mg} \mathrm{C}+\mathrm{AJ}$ & 996.86 & 190.32 & -44.05 & 156.02 & -28.51 & 146.07 & \\
\hline & $10.0 \mathrm{mg} \mathrm{C}+\mathrm{AJ}$ & 1075.93 & 320.12 & 5.37 & 290.82 & -63.92 & 332.90 & \\
\hline \multirow{6}{*}{$\begin{array}{l}\text { Delayed word recall - accuracy } \\
\text { (number correct) }\end{array}$} & Placebo & 2.76 & 2.11 & -0.72 & 2.15 & 0.10 & 2.48 & \\
\hline & $75.0 \mathrm{mg} \mathrm{C}$ & 3.56 & 2.25 & -1.18 & 2.72 & -0.52 & 1.98 & \\
\hline & $75.0 \mathrm{mg} \mathrm{C}+\mathrm{AJ}$ & 3.50 & 2.09 & -0.76 & 2.50 & -0.66 & 2.78 & \\
\hline & $37.5 \mathrm{mg} \mathrm{C}+\mathrm{AJ}$ & 3.80 & 2.42 & -0.96 & 2.96 & -0.66 & 2.76 & \\
\hline & $20.0 \mathrm{mg} \mathrm{C}+\mathrm{AJ}$ & 3.16 & 1.79 & -0.64 & 1.98 & -0.50 & 2.34 & \\
\hline & $10.0 \mathrm{mg} \mathrm{C}+\mathrm{AJ}$ & 3.36 & 1.77 & -1.26 & 2.08 & -0.42 & 1.97 & \\
\hline \multirow{6}{*}{$\begin{array}{l}\text { Delayed word recall - errors } \\
\text { (number incorrect) }\end{array}$} & Placebo & 0.36 & 0.64 & 0.36 & 1.25 & 0.24 & 1.09 & \\
\hline & $75.0 \mathrm{mg} \mathrm{C}$ & 0.64 & 0.95 & -0.20 & 0.96 & 0.16 & 1.11 & \\
\hline & $75.0 \mathrm{mg} \mathrm{C}+\mathrm{AJ}$ & 0.28 & 0.61 & 0.28 & 0.89 & 0.44 & 0.65 & \\
\hline & $37.5 \mathrm{mg} \mathrm{C}+\mathrm{AJ}$ & 0.68 & 1.44 & -0.28 & 0.98 & -0.40 & 1.12 & \\
\hline & $20.0 \mathrm{mg} \mathrm{C}+\mathrm{AJ}$ & 0.36 & 0.57 & 0.00 & 0.58 & 0.08 & 0.86 & \\
\hline & $10.0 \mathrm{mg} \mathrm{C}+\mathrm{AJ}$ & 0.44 & 0.92 & 0.08 & 0.70 & 0.08 & 0.70 & \\
\hline
\end{tabular}

$C$, caffeine; $C+A J$, apple extract with added caffeine

a 2 Beverages (test beverage compared to placebo) X 3 Times (baseline, post-beverage 1, and post-beverage 2)

${ }^{\mathrm{b}} R V I P$ rapid visual information processing test

cognitive tasks $(p=.01)$ but the effect was statistically insignificant.

$10 \mathrm{mg}$ CAF + Apple Extract The Group x Time interactions for $10 \mathrm{mg}$ CAF + apple extract effects on cognition were all statistically insignificant but one was in the expected direction - the increased total number of attempted serial 7 subtractions $(p=.027)$.

\section{Mood Outcomes}

Descriptive statistics for the mood outcomes are provided in Table 2. Statistically significant results for the mood outcomes are summarized below, and outcomes not mentioned below were not statistically significant.

Positive Control (75 mg CAF) Similar to the effects on cognitive performance, the positive control had the largest and most widespread effects on mood of all the evaluated beverages.
The largest effects were found in alertness measured using a single item visual analog scale (VAS) included within the CDB. There was a significant Group $\mathrm{x}$ Time interaction for $75 \mathrm{mg} \operatorname{CAF}\left(\eta_{p}^{2}=.235, p<.001\right)$ and post hoc tests showed alertness was increased compared to baseline at the first and second post-beverage time points. Alertness increased by 24.2 $( \pm 20.7)$ and $18.21( \pm 20.68)$ raw score units at post-beverage time points 1 and 2, respectively. Additionally, there was a significant Group $\mathrm{x}$ Time interaction for the positive control beverage for alertness measured using the single item BondLader $\left(\eta_{p}{ }^{2}=.174, p<.001\right)$. Post hoc tests found that BondLader alertness was increased compared to baseline at both post-beverage time points, with increases of $17.6( \pm 17.2)$ and $13.1( \pm 17.3)$ raw score units at post-beverage time 1 and time 2 , respectively.

There was also a significant Group x Time interaction for $75 \mathrm{mg}$ CAF for feelings of mental fatigue measured using a single item VAS $\left(\eta_{p}{ }^{2}=.131, p=.002\right)$. Post hoc tests showed that mental fatigue was decreased at both the first and second 
Table 2 Mean $( \pm \mathrm{SD})$ scores for mood and motivation across beverage conditions and time, and interaction significance

\begin{tabular}{|c|c|c|c|c|c|c|c|c|}
\hline & & \multicolumn{2}{|c|}{ Baseline } & \multicolumn{2}{|c|}{$\begin{array}{l}\text { Post } 1 \\
\text { Change from BL }\end{array}$} & \multicolumn{2}{|c|}{$\begin{array}{l}\text { Post } 2 \\
\text { Change from BL }\end{array}$} & \multirow[t]{2}{*}{$\begin{array}{l}\text { Beverage } \mathrm{x} \text { Time } \\
p \text { value }\end{array}$} \\
\hline & & Value & $\mathrm{SD}$ & Value & SD & Value & SD & \\
\hline \multirow{6}{*}{$\begin{array}{l}\text { Mental Fatigue } \\
\text { (VAS within CDB) }\end{array}$} & Placebo & 44.53 & 21.10 & 0.38 & 13.10 & 1.90 & 19.67 & \\
\hline & $75.0 \mathrm{mg} \mathrm{C}$ & 49.93 & 22.86 & -17.10 & 21.67 & -12.42 & 22.16 & .002 \\
\hline & $75.0 \mathrm{mg} \mathrm{C}+\mathrm{AJ}$ & 47.89 & 21.37 & -12.51 & 17.57 & -7.29 & 21.20 & \\
\hline & $37.5 \mathrm{mg} \mathrm{C}+\mathrm{AJ}$ & 50.06 & 18.16 & -14.36 & 18.33 & -11.81 & 19.29 & .004 \\
\hline & $20.0 \mathrm{mg} \mathrm{C}+\mathrm{AJ}$ & 44.76 & 18.94 & -2.82 & 17.03 & 2.4 & 17.95 & \\
\hline & $10.0 \mathrm{mg} \mathrm{C}+\mathrm{AJ}$ & 50.64 & 19.38 & -6.75 & 21.45 & -5.60 & 25.20 & \\
\hline \multirow{6}{*}{$\begin{array}{l}\text { Alertness } \\
\text { (VAS within CDB) }\end{array}$} & Placebo & 47.9 & 21.26 & -1.22 & 13.05 & -2.02 & 20.28 & \\
\hline & $75.0 \mathrm{mg} \mathrm{C}$ & 40.48 & 19.43 & 24.24 & 20.69 & 18.21 & 20.68 & $<.001$ \\
\hline & $75.0 \mathrm{mg} \mathrm{C}+\mathrm{AJ}$ & 43.12 & 20.77 & 15.15 & 19.14 & 11.56 & 18.72 & .002 \\
\hline & $37.5 \mathrm{mg} \mathrm{C}+\mathrm{AJ}$ & 42.77 & 19.52 & 15.23 & 20.41 & 14.01 & 19.13 & .001 \\
\hline & $20.0 \mathrm{mg} \mathrm{C}+\mathrm{AJ}$ & 48.46 & 18.54 & 2.62 & 17.23 & -1.86 & 16.84 & \\
\hline & $10.0 \mathrm{mg} \mathrm{C}+\mathrm{AJ}$ & 43.08 & 17.52 & 8.93 & 21.58 & 5.64 & 21.48 & \\
\hline \multirow{6}{*}{$\begin{array}{l}\text { Physical energy } \\
\text { (EFS-State scale) }\end{array}$} & Placebo & 169.35 & 69.67 & 5.10 & 34.38 & -1.10 & 44.03 & \\
\hline & $75.0 \mathrm{mg} \mathrm{C}$ & 145.93 & 66.42 & 43.74 & 59.54 & 37.00 & 60.47 & \\
\hline & $75.0 \mathrm{mg} \mathrm{C}+\mathrm{AJ}$ & 158.33 & 64.89 & 30.46 & 43.24 & 30.91 & 48.06 & \\
\hline & $37.5 \mathrm{mg} \mathrm{C}+\mathrm{AJ}$ & 160.33 & 62.15 & 29.87 & 45.11 & 30.23 & 49.39 & \\
\hline & $20.0 \mathrm{mg} \mathrm{C}+\mathrm{AJ}$ & 167.53 & 62.00 & 7.64 & 35.00 & 6.29 & 33.78 & \\
\hline & $10.0 \mathrm{mg} \mathrm{C}+\mathrm{AJ}$ & 146.68 & 63.49 & 21.66 & 46.08 & 11.18 & 46.57 & \\
\hline \multirow{6}{*}{$\begin{array}{l}\text { Physical fatigue } \\
\text { (EFS-State scale) }\end{array}$} & Placebo & 108.74 & 70.05 & 3.33 & 27.06 & 9.64 & 50.35 & \\
\hline & $75.0 \mathrm{mg} \mathrm{C}$ & 133.42 & 71.11 & -46.99 & 67.36 & -37.67 & 65.24 & .001 \\
\hline & $75.0 \mathrm{mg} \mathrm{C}+\mathrm{AJ}$ & 117.55 & 62.21 & -31.20 & 53.60 & -32.72 & 54.26 & .003 \\
\hline & $37.5 \mathrm{mg} \mathrm{C}+\mathrm{AJ}$ & 123.81 & 56.89 & -30.05 & 55.39 & -27.68 & 58.89 & \\
\hline & $20.0 \mathrm{mg} \mathrm{C}+\mathrm{AJ}$ & 108.18 & 62.47 & -10.04 & 38.79 & -7.30 & 39.26 & \\
\hline & $10.0 \mathrm{mg} \mathrm{C}+\mathrm{AJ}$ & 128.00 & 66.60 & -26.53 & 67.81 & -21.93 & 66.33 & \\
\hline \multirow{6}{*}{$\begin{array}{l}\text { Mental energy } \\
\text { (EFS-State scale) }\end{array}$} & Placebo & 150.27 & 71.25 & 6.63 & 41.61 & 1.67 & 62.44 & \\
\hline & $75.0 \mathrm{mg} \mathrm{C}$ & 127.57 & 65.35 & 50.18 & 56.76 & 40.27 & 67.29 & \\
\hline & $75.0 \mathrm{mg} \mathrm{C}+\mathrm{AJ}$ & 139.77 & 67.54 & 31.61 & 56.67 & 27.15 & 60.81 & \\
\hline & $37.5 \mathrm{mg} \mathrm{C}+\mathrm{AJ}$ & 134.75 & 55.54 & 39.22 & 54.84 & 38.19 & 59.72 & \\
\hline & $20.0 \mathrm{mg} \mathrm{C}+\mathrm{AJ}$ & 140.57 & 63.11 & 7.51 & 41.19 & 2.99 & 39.40 & \\
\hline & $10.0 \mathrm{mg} \mathrm{C}+\mathrm{AJ}$ & 127.85 & 61.81 & 21.26 & 60.91 & 4.92 & 66.58 & \\
\hline \multirow{6}{*}{$\begin{array}{l}\text { Mental fatigue } \\
\text { (EFS-State scale) }\end{array}$} & Placebo & 128.69 & 68.70 & -1.78 & 38.46 & 1.77 & 52.87 & \\
\hline & $75.0 \mathrm{mg} \mathrm{C}$ & 155.03 & 71.10 & -55.02 & 63.20 & -40.33 & 68.07 & .002 \\
\hline & $75.0 \mathrm{mg} \mathrm{C}+\mathrm{AJ}$ & 138.28 & 65.13 & -26.41 & 62.52 & -30.848 & 63.58 & \\
\hline & $37.5 \mathrm{mg} \mathrm{C}+\mathrm{AJ}$ & 154.30 & 56.06 & -42.58 & 59.06 & -40.56 & 62.22 & .005 \\
\hline & $20.0 \mathrm{mg} \mathrm{C}+\mathrm{AJ}$ & 136.65 & 65.08 & -9.75 & 41.18 & -3.45 & 42.35 & \\
\hline & $10.0 \mathrm{mg} \mathrm{C}+\mathrm{AJ}$ & 149.68 & 60.87 & -25.75 & 76.28 & -14.10 & 74.56 & \\
\hline \multirow{6}{*}{$\begin{array}{l}\text { Alertness } \\
\text { (Bond-Lader) }\end{array}$} & Placebo & 52.39 & 20.21 & 0.35 & 11.75 & -0.14 & 16.58 & \\
\hline & $75.0 \mathrm{mg} \mathrm{C}$ & 47.56 & 17.98 & 17.61 & 17.2 & 13.06 & 17.34 & $<.001$ \\
\hline & $75.0 \mathrm{mg} \mathrm{C}+\mathrm{AJ}$ & 51.63 & 19.40 & 8.00 & 14.32 & 5.96 & 16.22 & \\
\hline & $37.5 \mathrm{mg} \mathrm{C}+\mathrm{AJ}$ & 47.10 & 17.17 & 12.77 & 18.41 & 13.26 & 17.21 & .004 \\
\hline & $20.0 \mathrm{mg} \mathrm{C}+\mathrm{AJ}$ & 54.13 & 15.58 & -0.23 & 11.56 & -2.77 & 13.84 & \\
\hline & $10.0 \mathrm{mg} \mathrm{C}+\mathrm{AJ}$ & 48.54 & 17.25 & 6.37 & 19.25 & 4.71 & 19.45 & \\
\hline \multirow{4}{*}{$\begin{array}{l}\text { Calmness } \\
\text { (Bond-Lader) }\end{array}$} & Placebo & 67.99 & 12.92 & -4.00 & 9.51 & -6.39 & 12.19 & \\
\hline & $75.0 \mathrm{mg} \mathrm{C}$ & 67.16 & 15.66 & -10.42 & 19.31 & -9.38 & 17.33 & \\
\hline & $75.0 \mathrm{mg} \mathrm{C}+\mathrm{AJ}$ & 69.12 & 13.45 & -6.25 & 11.06 & -10.28 & 14.30 & \\
\hline & $37.5 \mathrm{mg} \mathrm{C}+\mathrm{AJ}$ & 65.81 & 12.11 & -6.46 & 17.33 & -7.11 & 18.29 & \\
\hline
\end{tabular}


Table 2 (continued)

\begin{tabular}{|c|c|c|c|c|c|c|c|c|}
\hline & & \multicolumn{2}{|c|}{ Baseline } & \multicolumn{2}{|c|}{$\begin{array}{l}\text { Post } 1 \\
\text { Change from } \mathrm{BL}\end{array}$} & \multicolumn{2}{|c|}{$\begin{array}{l}\text { Post } 2 \\
\text { Change from BL }\end{array}$} & \multirow[t]{2}{*}{$\begin{array}{l}\text { Beverage } \mathrm{x} \text { Time } \\
p \text { value }\end{array}$} \\
\hline & & Value & SD & Value & SD & Value & SD & \\
\hline \multirow{8}{*}{$\begin{array}{l}\text { Contentedness } \\
\text { (Bond-Lader) }\end{array}$} & $20.0 \mathrm{mg} \mathrm{C}+\mathrm{AJ}$ & 64.82 & 15.52 & -4.60 & 12.72 & -4.74 & 14.45 & \\
\hline & $10.0 \mathrm{mg} \mathrm{C}+\mathrm{AJ}$ & 65.33 & 14.10 & -4.26 & 14.16 & -3.94 & 12.64 & \\
\hline & Placebo & 65.64 & 16.91 & -0.29 & 4.33 & -0.17 & 10.38 & \\
\hline & $75.0 \mathrm{mg} \mathrm{C}$ & 64.64 & 15.97 & 5.48 & 10.80 & 3.01 & 10.77 & \\
\hline & $75.0 \mathrm{mg} \mathrm{C}+\mathrm{AJ}$ & 66.60 & 15.93 & 2.74 & 10.44 & 1.56 & 9.40 & \\
\hline & $37.5 \mathrm{mg} \mathrm{C}+\mathrm{AJ}$ & 66.28 & 17.39 & 5.79 & 12.36 & 5.67 & 12.13 & \\
\hline & $20.0 \mathrm{mg} \mathrm{C}+\mathrm{AJ}$ & 67.11 & 13.97 & -0.55 & 3.69 & -3.28 & 6.69 & \\
\hline & $10.0 \mathrm{mg} \mathrm{C}+\mathrm{AJ}$ & 63.06 & 16.57 & 3.39 & 15.03 & 2.66 & 14.10 & \\
\hline
\end{tabular}

$C$, caffeine; $C+A J$, apple extract with added caffeine

a 2 Beverages (test beverage compared to placebo) X 3 Times (baseline, post-beverage 1, and post-beverage 2)

post-beverage time points, with mental fatigue decreasing by $17.1( \pm 21.7)$ and $12.4( \pm 22.2)$ raw score units, respectively.

Significant Group x Time interactions for $75 \mathrm{mg} C A F$ were found for feelings of mental fatigue $\left(\eta_{p}^{2}=.135, p=.002\right)$ measured using 3-item fatigue sub-scale of Mental and Physical State Fatigue Scale (EFS-State scale). Post hoc tests showed that mental fatigue was decreased compared to baseline at both post-beverage time points.

The positive control beverage also significantly decreased feelings of physical fatigue $\left(\eta_{p}^{2}=.150, p=.001\right)$ measured using the EFS-State measures. Post hoc tests showed that physical fatigue was decreased compared to baseline at both post-beverage time points.

75 mg CAF + Apple Extract There was a significant Group x Time interaction for $75 \mathrm{mg} C A F+$ apple extract for decreased feelings of physical fatigue $\left(\eta_{p}{ }^{2}=.125, p=.003\right)$ measured using the EFS-State scales. Increased feelings of energy feelings of physical energy were in the expected direction but smaller in magnitude $(p=.016)$. Post hoc tests showed that feelings of physical fatigue were lower at both post-beverage time points compared to baseline. Decreases in mental fatigue measured with single item VAS were not significant $(p=.031)$.

$37.5 \mathrm{mg}$ CAF + Apple Extract There was a significant Group $\mathrm{x}$ Time interaction for $37.5 \mathrm{mg} C A F+$ apple extract for alertness measured using both the single item VAS $\left(\eta_{p}{ }^{2}=.136\right.$, $p=.001)$ and the Bond-Lader mood scale $\left(\eta_{p}{ }^{2}=.123\right.$, $p=.004)$. Post hoc tests showed that alertness was increased at both post-beverage time points for both measures. Single item VAS alertness increased $15.2( \pm 20.4)$ and $14.0( \pm 19.1)$ raw score units at time points 1 and 2, respectively. BondLader alertness increased $12.8( \pm 18.4)$ and $13.3( \pm 17.2)$ raw score unites at time points 1 and 2 , respectively.
There was a significant Group $\mathrm{x}$ Time interaction for $37.5 \mathrm{mg} \mathrm{CAF}+$ apple extract for mental fatigue measured with the single item VAS $\left(\eta_{p}{ }^{2}=.108, p=.004\right)$ and the EFSState scale $\left(\eta_{p}{ }^{2}=.111, p=.005\right)$. Post hoc tests showed that mental fatigue was decreased at both post-beverage time points when measured using both scales.

None of the significant Group $\mathrm{x}$ Time interactions for the cognition, mood, or motivation variables became statistically insignificant when typical caffeine consumption was added to the model as a covariate, supporting that the results were not explained by self-reported typical caffeine consumption.

\section{Discussion}

The results of the current study demonstrate that acute consumption of a beverage containing $75 \mathrm{mg}$ of caffeine plus apple extract can improve the speed of information processing, specifically serial seven subtraction information, while $37.5 \mathrm{mg}$ of caffeine plus apple extract improved feelings of alertness and mental fatigue.

We hypothesized that in comparison to a colored and flavored placebo beverage without apple extract or caffeine, cognitive performance, mood, and motivation would improve after consuming apple extract containing added caffeine in low doses of 10 and $20 \mathrm{mg}$. This hypothesis was not confirmed because the beverages with either 10 or $20 \mathrm{mg}$ of caffeine had no influence on cognitive performance. It has been shown previously that $2.5 \mathrm{mg}$ of caffeine delivered in berry extract resulted in worse cognitive performance, while $5 \mathrm{mg}$ of caffeine in fruit extract resulted in mixed effects on cognitive performance (Haskell et al. 2008). The null cognitive findings for $10 \mathrm{mg}$ caffeine failed to support positive findings presented in abstract form previously and found that $10 \mathrm{mg}$ of caffeine delivered in a fruit extract improved cognitive 
performance at 1, 3, 6, and $9 \mathrm{~h}$ post ingestion. Another study examining a low amount of caffeine, $12.5 \mathrm{mg}$ delivered via capsule form, found improvements in simple reaction time at 77-97 min post dose in both high and low consumers of caffeine, while improvements in RVIP were found in high consumers of caffeine only (Smit and Rogers 2000). The present sample included two individuals who reported high daily caffeine consumption of 689 and $741 \mathrm{mg}$ per day. In short, it appears that the cognitive and motivation effects of lower amounts of caffeine combined with an apple extract drink are small and variable within the present paradigm which involved relatively long and boring testing sessions. Anecdotally, several participants confirmed the general difficulty of the testing session by indicating immediately after the final testing session they were pleased to not have to endure any additional testing sessions.

As expected, a drink without apple extract and containing $75 \mathrm{mg}$ of caffeine (the positive control) was effective at improving cognitive performance in various domains including total number of serial 3 and serial 7 subtractions attempted at post-beverage times 1 and 2, and the number of serial 3 and serial 7 correct responses at both post-beverage times. A comprehensive review by Einother et al. (Einother and Giesbrecht 2013) examining the effects of caffeine on attention tasks found that RVIP accuracy was generally improved with doses of caffeine ranging from 50 to $150 \mathrm{mg}$, while higher doses, 150-450 mg, were associated with faster RVIP response times. The current RVIP accuracy findings were in the direction consistent with the prior research but not strong enough to be statistically significant after correcting for multiple statistical tests ( $p=.026$ did not reach the $p \leq .005$ threshold).

A dose-dependent effect on cognition was rejected for most variables and supported for one. No significant effects were found for the apple extract beverage containing $37.5 \mathrm{mg}$ of caffeine while the apple extract beverage containing $75 \mathrm{mg}$ caffeine did show a significant effect for the number of serial 7 subtractions attempted. This outcome was not due to greater motivation in the higher caffeine condition. Thus, the greater number of serial 7 subtractions is likely due to an increase in information processing speed. Nevertheless, the apple extract beverage containing $37.5 \mathrm{mg}$ of caffeine had a greater number of small magnitude statistically insignificant changes that, if they were liberally interpreted, may provide general support of a small degree of enhanced cognitive performance. At best there appears to be subtle effects on cognition of the apple extract beverage containing $37.5 \mathrm{mg}$ of caffeine. This could be because the caffeine and apple polyphenols have interactive effects on brain circuits that underlie several processes involved in cognitive performance, including the perceived motivation to perform the cognitive work. Due to caffeine's inhibitory effects on adenosine $\mathrm{A}_{2 \mathrm{a}}$ receptors which are located in widespread brain areas, caffeine ingestion increases the release of neurotransmitters, such as norepinephrine and dopamine, while simultaneously decreasing cerebral blood flow (Kennedy and Wightman 2011). Polyphenol supplementation, though less well understood, can increase cerebral blood flow through nitric oxide augmentation (Wightman et al. 2014).

Currently, there appears to be only one study available that examined the effects of apples alone on cognitive outcomes (Bondonno et al. 2014). Bondonno et al. (Bondonno et al. 2014) used apples containing $364 \mathrm{mg}$ of polyphenols (quercetin glycosides and (-)-epicatechin) compared to a control with a low concentration of apple polyphenols. As polyphenol supplementation alone did not significantly change cognitive performance or mood, perhaps polyphenols consumed alone and in moderate doses are not effective and need to be combined with other ingredients at the proper dosages to maximize effectiveness on cognitive outcomes.

With regard to the results for the mood measures, the strongest effects were found for the beverage with $37.5 \mathrm{mg}$ caffeine plus apple extract. Compared with the placebo, beverages containing 20 and $10 \mathrm{mg}$ of caffeine combined with apple extract had no effect on mood, the beverage containing $75 \mathrm{mg}$ caffeine plus apple extract improved feelings of physical fatigue, and the beverage containing $37.5 \mathrm{mg}$ caffeine plus apple extract improved two measures of feelings of alertness and two measures of mental fatigue.

There are no identical studies in the literature that measured mood, so direct comparisons to the present findings are not possible. Apple consumption containing $364 \mathrm{mg}$ of polyphenols (184 mg quercetin glycosides and $180 \mathrm{mg}(-)$-epicatechin) did not produce positive or negative changes in alertness, calmness, or contentedness 150 -min post-consumption (Bondonno et al. 2014). However, reduced ratings of mental fatigue after performing cognitive tests have been realized 1.5 to $2.5 \mathrm{~h}$ after the consumption of a drink containing $520 \mathrm{mg}$ of cocoa flavanols (Scholey et al. 2010). Specifically, attenuations in mental fatigue were found at 10,20,40, and $60 \mathrm{~min}$ with $520 \mathrm{mg}$ cocoa flavanol consumption, but not $994 \mathrm{mg}$.

A review of the bioavailability of polyphenols in humans, specifically examining quercetin, which is found in apples, found that large inter-individual variability in plasma concentrations of quercetin occurs with polyphenol supplementation, leading to the possibility of "high absorbers" and "low absorbers" of polyphenols (Manach et al. 2005) which can occur in part due to variation in the bi-directional relationships between polyphenols and intestinal microbiota (Fraga et al. 2019) Kawabata et al. 2019). This may translate into "responders" and "non-responders" to polyphenol supplementation and increase the variability in the ability of polyphenols to affect cognition, motivation, and mood outcomes. As the response to caffeine can also vary among individuals, the interactive effects of one's predisposition for "reception" of polyphenols and caffeine may affect polyphenol's effects on cognitive and mood outcomes. Our analysis, however, failed to 
support self-reported typical caffeine consumption as a variable that explained variation in the results.

Finally, when examining the current literature on both caffeine and polyphenol effects on cognition and mood, it is challenging to draw general conclusions and make direct comparisons among results in light of the methodological differences among studies. These differences include varying sample characteristics (e.g., younger people may better absorb nutrients compared to older adults, low apple or caffeine consumers may have greater responses than those with higher consumption patterns, fatigued people may show larger effects than those who feel energetic), varying doses of both caffeine and polyphenols, varying types of polyphenols used for supplementation, varying time periods between supplementation and testing, and varying cognitive tests and task times employed to assess cognitive ability. Also, most studies of this type have not reported the reliability of the outcome measures for which test beverage effects were not observed even though low reliability can occur and its presence accounts for the lack of test beverage effect.

All studies have some limitations. Due to previous findings on apple extract mood effects, we did not include a control arm testing apple extract without caffeine, and this may be a major limitation in determining the psychological effects of caffeine combined with apple extract. While participants were asked to refrain from eating food and beverages containing apples, research staff could only suggest this and were unable to mandate it for participation. Avoiding other flavanol rich food and beverage items would also have been advantageous for the present investigation, but without providing standardized pretest meals and beverages for all participants at all testing visits, this is difficult to monitor and enforce. The unknown and/or variable washout period of flavanols also complicates nutrition prior to testing. Participants also selfreported food and beverage intake and compliance with previsit requirements, including avoidance of exercise. Additionally, we did not use any objective measurement of caffeine avoidance, such as salivary assessment. Finally, most participants were female and all were generally young and healthy; therefore, the results may not be generalizable to other populations. For example, a recent investigation involving only male participants found the consumption of a high dose of cocoa (1052 mg total polyphenols), compared to a low dose (143 mg), improved cognitive performance when cognitive demand was high (Gratton et al. 2020).

In conclusion, the current study is the first to examine the combined effects of varying amounts of caffeine and apple polyphenols on cognition and mood. The present study shows that moderate doses of caffeine combined with apple extract are effective at improving cognitive outcomes, especially serial seven subtraction, and mood responses to the tasks, including alertness and mental and physical fatigue. Very low doses of caffeine (10 to $20 \mathrm{mg}$ ) combined with apple extract are not consistently associated with improvements in cognitive, motivation, or mood outcomes.

\section{Compliance with Ethical Standards}

All procedures performed in this study were consistent with the 1964 Helsinki declaration and its later amendments or comparable ethical standards. The study was approved by the University Institutional Review Committee for human research participants and informed consent was obtained from all participants. The work described here was supported by PepsiCo, Inc. through the provision of the beverages and a grant to the University of Georgia for the costs of conducting the research. A PepsiCo Inc. employee, Alissa Wilson, contributed to the conception and design of the study. The other authors have no conflicts of interest. The views expressed in this article are those of the authors and do not necessarily reflect the position or policy of PepsiCo, Inc. The datasets generated during and/or analyzed during the current study are available from the corresponding author on reasonable request.

Open Access This article is licensed under a Creative Commons Attribution 4.0 International License, which permits use, sharing, adaptation, distribution and reproduction in any medium or format, as long as you give appropriate credit to the original author(s) and the source, provide a link to the Creative Commons licence, and indicate if changes were made. The images or other third party material in this article are included in the article's Creative Commons licence, unless indicated otherwise in a credit line to the material. If material is not included in the article's Creative Commons licence and your intended use is not permitted by statutory regulation or exceeds the permitted use, you will need to obtain permission directly from the copyright holder. To view a copy of this licence, visit http://creativecommons.org/licenses/by/4.0/.

\section{References}

Alonso-Salces, R. M., Barranco, A., Abad, B., Berrueta, L. A., Gallo, B., \& Vicente, F. (2004). Polyphenolic profiles of basque cider apple cultivars and their technological properties. Journal of Agricultural and Food Chemistry, 52(10), 2938-2952.

Bondonno, C. P., Downey, L. A., Croft, K. D., Scholey, A., Stough, C., Yang, X., Considine, M. J., Ward, N. C., Puddey, I. B., Swinny, E., Mubarak, A., \& Hodgson, J. M. (2014). The acute effect of flavonoid-rich apples and nitrate-rich spinach on cognitive performance and mood in healthy men and women. Food \& Function, $5(5), 849-858$.

Chen, C., \& Blumberg, J. B. (2008). Are there age related changes in flavonoid bioavailability? In Phytochemicals aging and health (pp. 19-31). Boca Raton: CRC Press.

D’Amico, E. J., Neilands, T. B., \& Zambarano, R. (2001). Power analysis for multivariate and repeated measures designs: a flexible approach using the spss manova procedure. Behavior Research Methods, Instruments, \& Computers, 33(4), 479-484.

Einother, S. J., \& Giesbrecht, T. (2013). Caffeine as an attention enhancer: reviewing existing assumptions. Psychopharmacology, 225(2), 251-274.

Field, D. T., Williams, C. M., \& Butler, L. T. (2011). Consumption of cocoa flavanols results in an acute improvement in visual and cognitive functions. Physiology \& Behavior, 103(3-4), 255-260.

Fisher, N. D., Sorond, F. A., \& Hollenberg, N. K. (2006). Cocoa flavanols and brain perfusion. Journal of Cardiovascular Pharmacology, 47(Suppl 2), S210-S214. 
Fraga, C. G., Croft, K. D., Kennedy, D. O., \& Tomás-Barberán, F. A. (2019). The effects of polyphenols and other bioactives on human health. Food \& Function, 10, 514-528.

Fredholm, B. B. (2012). How drugs can stimulate psychic functions using caffeine as an example. European Review, 20(3), 316-323.

Gibbs, M. E., Hutchinson, D. S., \& Summers, R. J. (2008). Role of betaadrenoceptors in memory consolidation: Beta3-adrenoceptors act on glucose uptake and beta2-adrenoceptors on glycogenolysis. Neuropsychopharmacology, 33(10), 2384-2397.

Godin, G., \& Shephard, R. J. (1985). A simple method to assess exercise behavior in the community. Canadian Journal of Applied Sport Sciences, 10(3), 141-146.

Gonzalez de Mejia, E., \& Ramirez-Mares, M. V. (2014). Impact of caffeine and coffee on our health. Trends in Endocrinology and Metabolism, 25(10), 489-492.

Gratton, G., Weaver, S. R., Burley, C. V., Low, K. A., Maclin, E. L., Johns, P. W., Pham, Q. S., Lucas, A. J. E., Fabiani, M., \& Rendeiro, C. (2020). Dietary flavanols improve cerebral cortical oxygenation and cognition in healthy adults. Scientific Reports, 10, 19409.

Haskell, C. F., Kennedy, D., Milne, A., Wesnes, K., \& Scholey, A. B. (2008). Caffeine at levels found in decaffeinated beverages is behaviourally active. Appetitie, 50, 559.

Kahle, K., Kempf, M., Schreier, P., Scheppach, W., Schrenk, D., Kautenburger, T., Hecker, D., Huemmer, W., Ackermann, M., \& Richling, E. (2011). Intestinal transit and systemic metabolism of apple polyphenols. European Journal of Nutrition, 50(7), 507-522.

Kawabata, K., Yoshioka, Y., \& Terao, J. (2019). Role of intestinal microbiota in the bioavailability and physiological functions of dietary polyphenols. Molecules, 24(2), 370-395.

Kennedy, D. O., \& Wightman, E. L. (2011). Herbal extracts and phytochemicals: plant secondary metabolites and the enhancement of human brain function. Advances in Nutrition, 2(1), 32-50.

Khan, M., Elango, C., Ansari, M. A., Singh, I., \& Singh, A. K. (2007). Caffeic acid phenethyl ester reduces neurovascular inflammation and protects rat brain following transient focal cerebral ischemia. Journal of Neurochemistry, 102(2), 365-377.

Lamport, D. J., Dye, L., Wightman, J. D., \& Lawton, C. L. (2012). The effects of flavonoid and other polyphenol consumption on cognitive performance: a systematic research review of human experimental and epidemiological studies. Nutrition and Aging, 1(1), 5-25.

Manach, C., Williamson, G., Morand, C., Scalbert, A., \& Remesy, C. (2005). Bioavailability and bioefficacy of polyphenols in humans. I. Review of 97 bioavailability studies. The American Journal of Clinical Nutrition, 81(1 Suppl), 230S-242S.

Marder, M., Viola, H., Wasowski, C., Wolfman, C., Waterman, P. G., Medina, J. H., \& Paladini, A. C. (1996). Cirsiliol and caffeic acid ethyl ester, isolated from salvia guaranitica, are competitive ligands for the central benzodiazepine receptors. Phytomedicine, $3(1), 29-31$.
Maridakis, V., Herring, M. P., \& O’Connor, P. J. (2009). Sensitivity to change in cognitive performance and mood measures of energy and fatigue in response to differing doses of caffeine or breakfast. The International Journal of Neuroscience, 119(7), 975-994.

Marks, S. C., Mullen, W., \& Crozier, A. (2007). Flavonoid and chlorogenic acid profiles of english cider apples. Journal of the Science of Food and Agriculture, 87(4), 719-728.

O'Connor, P. J. (2006). Mental energy: developing a model for examining nutrition-related claims. Nutrition Reviews, 64(7 Pt 2), S2-S6.

Pase, M. P., Scholey, A. B., Pipingas, A., Kras, M., Nolidin, K., Gibbs, A., Wesnes, K., \& Stough, C. (2013). Cocoa polyphenols enhance positive mood states but not cognitive performance: a randomized, placebo-controlled trial. Journal of Psychopharmacology, 27(5), $451-458$.

Reed, J. A., \& Phillips, D. A. (2005). Relationships between physical activity and the proximity of exercise facilities and home exercise equipment used by undergraduate university students. Journal of American College Health, 53(6), 285-290.

Saarenhovi, Salo, P., Scheinin, M., Lehto, J., Lovró, Z., Tiihonen, K., Lehtinen, M. J., Junnila, J., Hasselwander, O., Tarpila, A., \& Raitakari, O. T. (2017). The effect of an apple polyphenol extract rich in epicatechin and flavan-3-ol oligomers on brachial artery flow-mediated vasodilatory function in volunteers with elevated blood pressure. Nutrition Journal, 16(1), 73.

Scholey, A. B., French, S. J., Morris, P. J., Kennedy, D. O., Milne, A. L., \& Haskell, C. F. (2010). Consumption of cocoa flavanols results in acute improvements in mood and cognitive performance during sustained mental effort. Journal of Psychopharmacology, 24(10), $1505-1514$.

Scholey, A., Downey, L. A., Ciorciari, J., Pipingas, A., Nolidin, K., Finn, M., Wines, M., Catchlove, S., Terrens, A., Barlow, E., Gordon, L., $\&$ Stough, C. (2012). Acute neurocognitive effects of epigallocatechin gallate (egcg). Appetite, 58(2), 767-770.

Smit, H. J., \& Rogers, P. J. (2000). Effects of low doses of caffeine on cognitive performance, mood and thirst in low and higher caffeine consumers. Psychopharmacology, 152(2), 167-173.

Wightman, E. L., Haskell, C. F., Forster, J. S., Veasey, R. C., \& Kennedy, D. O. (2012). Epigallocatechin gallate, cerebral blood flow parameters, cognitive performance and mood in healthy humans: a doubleblind, placebo-controlled, crossover investigation. Human Psychopharmacology, 27(2), 177-186.

Wightman, E. L., Reay, J. L., Haskell, C. F., Williamson, G., Dew, T. P., \& Kennedy, D. O. (2014). Effects of resveratrol alone or in combination with piperine on cerebral blood flow parameters and cognitive performance in human subjects: a randomised, double-blind, placebo-controlled, cross-over investigation. The British Journal of Nutrition, 112(2), 203-213.

Publisher's Note Springer Nature remains neutral with regard to jurisdictional claims in published maps and institutional affiliations. 